\title{
Stimulating Effect of Phenolic Compounds on Adventitious Bud Induction in Stem Segments and Seedlings of Torenia
}

\author{
Shizufumi TANIMOTo* and Takao ShImomura*
}

When long stem segments $(2 \mathrm{~cm}$ in length) of Torenia were cultured on medium containing cytokinin, adventitious buds were predominantly induced from the cut ends of the explants. When the segment was cultured on the medium with cytokinin and acetosyringon(AS), buds could be induced at the central part of the segment as well. It was noted that low concentration of AS as low as $0.001 \mu \mathrm{M}$ was effective. Furthermore, other phenolic compounds such as sinapic acid, ferulic acid, syringaldehyde and acetovanillone also promoted bud formation at the central part of the segment. In the central part of segment, the number of buds initiated seemed to be dependent on the concentrations of phenolic compounds. The largest number of buds per central part of the segment was obtained at $0.001 \mu \mathrm{M}$ of $\mathrm{AS}, 0.1 \mu \mathrm{M}$ of sinapic acid and $1 \mu \mathrm{M}$ of ferulic acid, syringaldehyde and acetovanillone. These phenolic compounds also promoted direct adventitious bud induction in the seedlings of Torenia. The above results suggested that these phenolic compounds or similar substance(s) produced by wounding, stimulated bud induction at the uninjured central part of the segment together with cytokinin.

Adventitious bud formation from organ segments in higher plants is thought to be regulated through the quantitative balance between auxin and cytokinin applied to the culture medium ${ }^{1)}$. In Petunia $^{2)}$ and Antirrhinum ${ }^{3}$, adventitious buds were induced when the ratio of cytokinin to auxin was high in the medium. However, we have previously demonstrated that adventitious bud initiation in Torenia stem segments was induced by only cytokinin, and was suppressed by auxin even at low concentrations ${ }^{4}$. In Torenia stem segments, adventitious buds could be induced by anaerobic treatment ${ }^{5)}$, calcium ionophore ${ }^{6)}$, cyclic $\mathrm{AMP}^{7)}$, phorbol ester ${ }^{8)}$ and spermidine ${ }^{9)}$. Therefore, this material, because of its high sensitivity to various stimuli, seemed to be a reasonable one for understanding the mechanisms of adventitious bud initiation.

The differences in response to cultural conditions between callus tissues and stem segments may arise from the wounding treatment which may affect adventitious bud induction in organ segments. In lily bulb-scale segments, the number of adventitious bulblets was increased by increasing of wounding area given to the segment ${ }^{10)}$. The cutting treatment seemed to produce some substance $(\mathrm{s})$ from destructed cells, such as phenylpropans and phytoalexins ${ }^{11)}$ that activated some metabolic processes including meristematic cell division, and accumulated in the wounding site.

In Solanaceae, it was generally known that phenolic compounds such as acetosyringon(AS), sinapic acid(SA), ferulic acid(FA), syringaldehyde(SAH) and acetovanillone( $\mathrm{AV}$ ), were produced when the plant was injured ${ }^{12)}$. It was reported that gene transfer to plant cells by Agrobacterium needed these phenolic compounds ${ }^{13)}$ which were induced by wounding. Some phenolic substances produced by cutting seemed to be involved in the bud induction from organ segments, as well. Therefore, we investigated the effects of phenolic compounds on adventitious bud initiation in Torenia long stem segments.

\footnotetext{
* Genetic Engineering Laboratory, Faculty of Agriculture, Saga University, Honjo, Saga 840, Japan
} 
When seedlings of Torenia were cultivated on the medium containing cytokinin, adventitious buds could be directly induced from hypocotyls of the seedlings ${ }^{14}$. This system was thought to be a useful means for understanding the effects of wounding. Therefore, we also examined the effects of phenolic compounds on direct adventitious bud induction in Torenia seedlings.

Plants of Torenia fornieri Lind. were grown under long-day photoperiod (16-h light 8-h dark, 500 $\left.\mu \mathrm{mol} \cdot \mathrm{m}^{-2} \cdot \mathrm{s}^{-1}\right)$ at constant temperature of $25 \pm 2^{\circ} \mathrm{C}$. Internodal segments $(2 \mathrm{~cm}$ in length $)$ taken from the upper parts of the stems were surface-sterilized with $\mathrm{NaOCl}$ solution (available chlorine, 1\%) for 20 min., rinsed in sterilized water, and used as explants.

Basal medium used was Murashige and Skoog's mineral salts and vitamins ${ }^{15)}$, containing $2 \%$ sucrose, $0.25 \%$ Gelrite(Merck) (hereafter referred to as MS medium), and $0.5 \mu \mathrm{M}$ of benzyladenine (BA), unless otherwise stated. Acetosyringon, SA, FA, SAH, and AV were sterilized through Millipore filter $(0.25 \mu \mathrm{m})$, and added to the medium.

The segments were put to the medium and cultured for 3 weeks. The cultures were maintained under 16 -h long-day photoperiod $\left(500 \mu \mathrm{mol} \cdot \mathrm{m}^{-2} \cdot \mathrm{s}^{-1}\right)$ at $25 \pm 2^{\circ} \mathrm{C}$.

After 3 weeks of culture, the cultured segments were divided into 8 parts $(2.5 \mathrm{~mm}$ in length), and then the number of buds was counted for every part from the apical to the basal end of the explants. At least 150 explants were used for each treatment. All experiments were repeated at least 3 times and the standard errors were calculated.

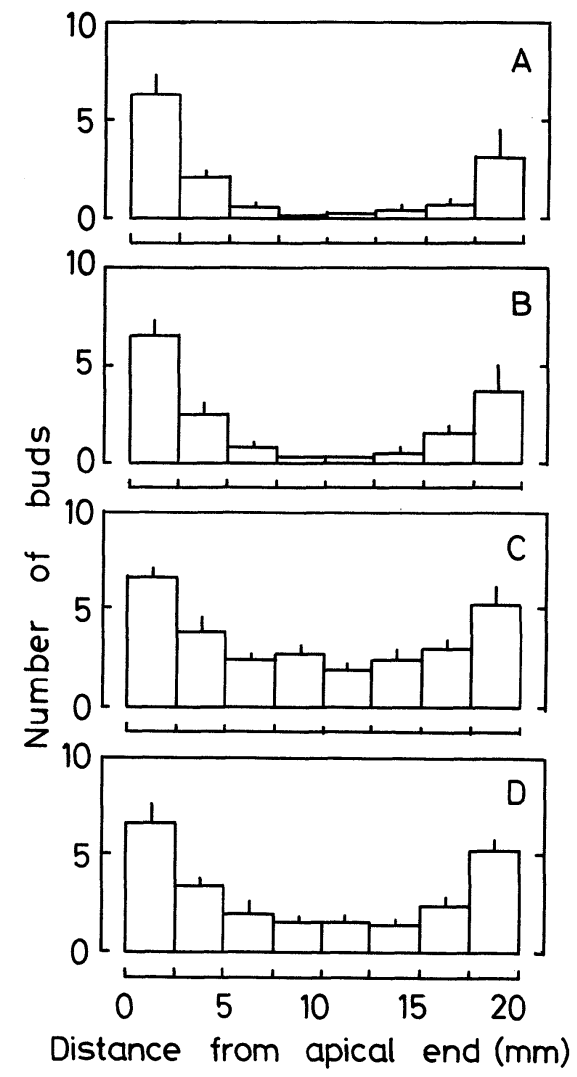

Fig. 1 Effect of acetosyringon(AS) on adventitious bud formation in Torenia stem segments. Long $(2 \mathrm{~cm}$ in length) stem segments were cultured for 3 weeks on the MS medium containing $0.5 \mu \mathrm{M}$ of BA, and divided into 8 parts $(2.5 \mathrm{~mm}$ in length), then the number of buds was counted every part from apical to basal end of the explants. The medium contained $0.5 \mu \mathrm{M}$ of $\mathrm{BA}$ and various concentrations of AS.

A; without AS, B; $0.0001 \mu \mathrm{M}$ of AS, C; $0.001 \mu \mathrm{M}$ of AS, D; $0.01 \mu \mathrm{M}$ of AS. 
Effects of phenolic compounds on adventitious bud induction from seedlings were examined as follows. Seeds of $T$. fournieri were sterilized with $\mathrm{NaOCl}$ solution (available chlorine, $1 \%$ ), rinsed in sterilized water and sown in MS medium containing $1 \mu \mathrm{M}$ of BA and various concentrations of

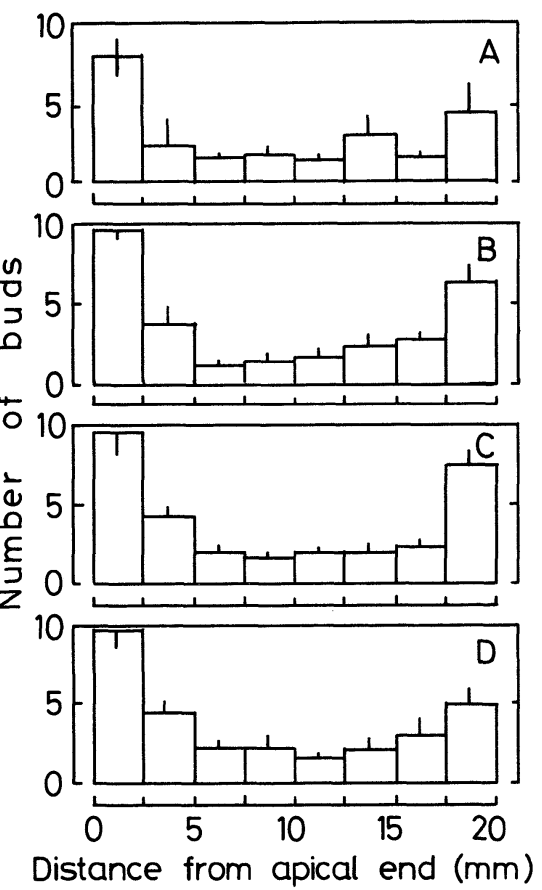

Fig. 2 Effect of phenolic compounds on adventitious bud formation in Torenia stem segments. The medium contained $0.5 \mu \mathrm{M}$ of $\mathrm{BA}$ and various kinds of phenolic compounds.

A; $0.1 \mu \mathrm{M}$ of sinapic acid, B; $1 \mu \mathrm{M}$ of ferulic acid, C; $1 \mu \mathrm{M}$ of syringaldehyde, D; $1 \mu \mathrm{M}$ of acetovanillone.

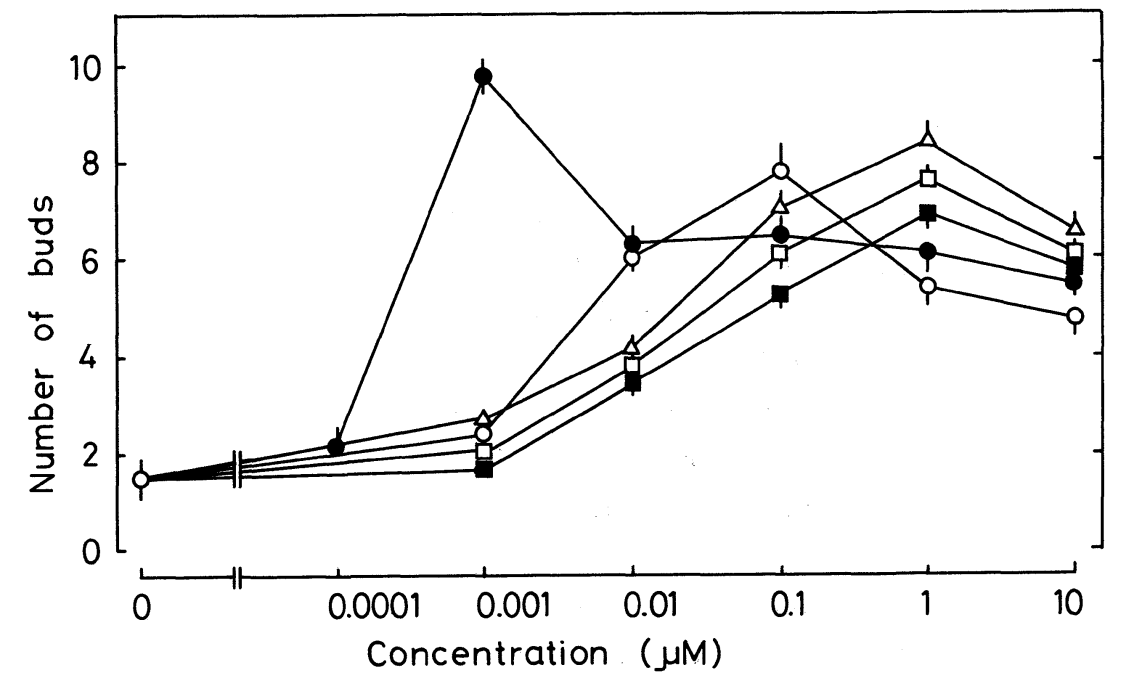

Fig. 3 Effect of phenolic compounds on number of adventitious buds in central part of Torenia stem segments.

The medium contained $0.5 \mu \mathrm{M}$ of BA and various kinds of phenolic compounds such as acetosyringon $(\bullet)$, sinapic $\operatorname{acid}(\bigcirc)$, ferulic $\operatorname{acid}(\boldsymbol{\square})$, syringaldehyde $(\square)$ and acetovanillone $(\triangle)$. After 3 weeks of culture, the number of adventitious buds per central part (5 to $15 \mathrm{~mm}$ from apical end) of segment was observed. 


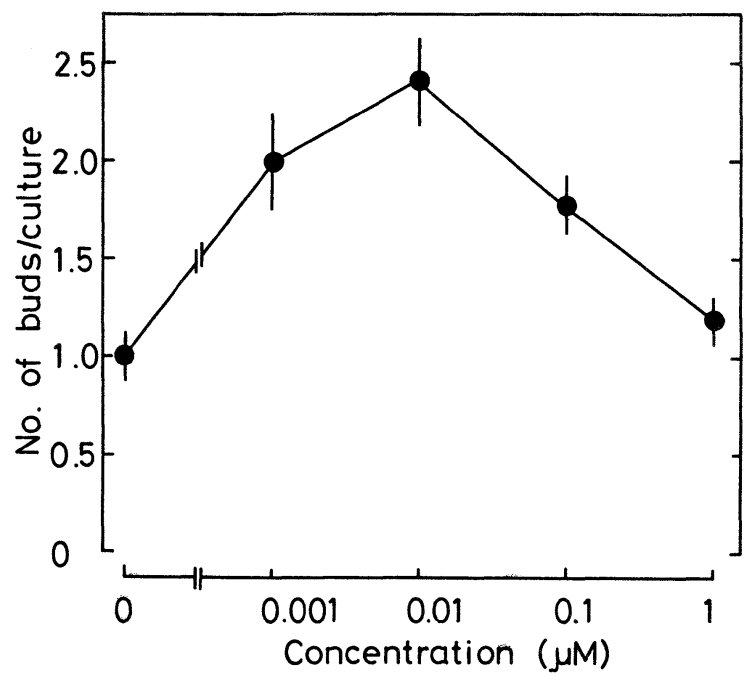

Fig. 4 Effect of acetosyringon on adventitious bud formation in Torenia seedlings.

The seeds were sown on the MS medium containing $1 \mu \mathrm{M}$ of BA and various concentrations of acetosyringon. After 5 weeks of culture, the number of adventitious buds per seedling was observed.

AS. After 5 weeks of culture, the number of adventitious buds per seedling was estimated. More than 100 seeds were used in each treatment. All experiments were repeated at least 3 times and the standard errors were calculated.

When long stem segments of Torenia were cultured on the medium containing cytokinin, few adventitious buds were induced at the central part of the explants ${ }^{16}$. On the other hand, more buds were initiated within the limits of $5 \mathrm{~mm}$ from additional wounding cite $^{16)}$.

As shown in Fig. 1, bud initiation at the central part of the explant was clearly stimulated by the application of AS at a concentration of more than $0.001 \mu \mathrm{M}($ Fig. 1-C and 1-D).

The effects of various concentrations of other phenolic compounds such as SA, FA, SAH and AV on adventitious bud formation were also examined. Bud formation at the central part of the explant was promoted by $0.1 \mu \mathrm{M}$ of SA(Fig. 2-A), $1 \mu \mathrm{M}$ of FA(Fig. 2-B), $1 \mu \mathrm{M}$ of SAH (Fig. 2-C) and $1 \mu \mathrm{M}$ of $\mathrm{AV}$ (Fig. 2-D).

The number of buds initiated in the central part ( 5 to $15 \mathrm{~mm}$ from apical end) of each segment was calculated. As shown in Fig. 3, the largest number of buds, 10 per central part of the segment, was obtained at $0.001 \mu \mathrm{M}$ of various AS concentrations. Similarly, application of $0.1 \mu \mathrm{M}$ of SA, $1 \mu \mathrm{M}$ of FA, SAH and AV were the most effective for bud induction.

When seedlings of Torenia were cultivated on the medium with AS, derect adventitious bud initiation was stimulated. Although 1 bud was initiated in the seedling cultivated on the medium containing $1 \mu \mathrm{M}$ of $\mathrm{BA}$, the simultaneous addition of $0.01 \mu \mathrm{M}$ of AS caused 2.4 buds per seedling (Fig. 4).

In tobacco callus, Lee and Skoog ${ }^{17)}$ reported that mono-hydroxy-substituted phenols were effective for bud induction. Rucker and Paupardin ${ }^{18)}$ described that various phenolic compounds promoted root formation in Jerusalem artichoke tuber explants.

The results obtained in this study suggested that, in Torenia stem segments and seedlings, not only cytokinin but also phenolic compounds produced by wounding stimulated adventitious bud initiation.

(Accepted October 21, 1995) 


\section{References}

1) Skoog, F., C. O. Miller, 1957. Symp. Soc. Exp. Biol., 11: 118-131.

2) Rao, P. S., V. A. Bapat, H. Harada, 1976. Z. Pflanzenphysiol., 80: 144-152.

3) Sangwan, R. S., H. Harada, 1975. J. Exp. Bot., 26: 868-881.

4) Tanimoto, S., H. Harada, 1984. J. Plant Physiol., 115: 11-18.

5) Tanimoto, S., H. Harada, 1983. Z. Pflanzenphysiol., 113: 85-90.

6) Tanimoto, S., H. Harada, 1986. Plant Cell Physiol., 27: 1-10.

7) Ishioka, N., S. Tanimoto, 1990. Plant Cell Physiol., 31: 91-97.

8) Ishioka, N., S. Tanimoto, 1992. Plant Tissue Culture Letters, 9: 86-89.

9) Tanimoto, S., Y. Matsubara, N. Ishioka, 1994. Plant Cell Physiol., 35: 1071-1077.

10) Ishioka, N., S. Tanimoto, 1990. Bull. Fac. Agr., Saga Univ., 69: 27-33.

11) Cruickshank, I. A. M., D. R. Perrin, 1968. Life Sci., 7: 449-458.

12) Stachel, S. E., E. Messens, M. Van Montagu, P. Zambryski, 1985. Nature, 318: 624-629.

13) Stachel, S. E., B. Timmerman, P. Zambryski, 1986. Nature, 322: 706-712.

14) Tanimoto, S., M. Sueishi, 1995. Plant Tissue Culture Letters, 12: 83-86.

15) Murashige, T., F. Skoog, 1962. Physiol. Plant., 15: 472-497.

16) Takeuchi, N., S. Tanimoto, H. Harada, 1985. J. Exp. Bot., 36: 841-847.

17) Lee, T. T., F. Skoog, 1965. Physiol. Plant., 18: 396-402.

18) Rucker, W., C. Paupardin, 1969. C. R. Acad. Sci. Paris Ser. D, 268: 1279-1281. 